Research Square
Preprints are preliminary reports that have not undergone peer review.

They should not be considered conclusive, used to inform clinical practice, or referenced by the media as validated information.

\title{
HDL Cholesterol Efflux Capacity and Concentration in Patients With Rheumatoid Arthritis: a Systematic Review and Meta-Analysis
}

Binbin Xie

Jinan university

Jiang He

Southern Medical University

Yong Liu

Anhui Medical University

Ting Liu

Jinan University

Chaoqun Liu ( $D$ Icqun1987@126.com)

Jinan University https://orcid.org/0000-0001-8322-3290

\section{Research}

Keywords: cholesterol efflux capacity, high density lipoprotein, meta-analysis, rheumatoid arthritis

Posted Date: August 24th, 2020

DOI: https://doi.org/10.21203/rs.3.rs-61508/v1

License: (c) (1) This work is licensed under a Creative Commons Attribution 4.0 International License. Read Full License 


\section{Abstract}

Background: HDL cholesterol efflux capacity has been proposed as an independent risk factor of cardiovascular diseases. However, the presented data has been less than convincing in RA. This meta-analysis aimed to identify whether CEC was impaired in RA or altered by drug therapy.

Methods: PubMed, Embase, and Cochrane library databases were searched to identify studies for CEC in RA patients. The primary outcomes were CEC, highdensity lipoprotein cholesterol and C-reactive protein.

Results: A total of 11 eligible articles including 6 observational and 5 intervention studies. Pooled results showed there was no significantly decrease in the CEC of RA patients comparing with the healthy controls (SMD: $-0.22,95 \% \mathrm{Cl}:-0.65$ to 0.20 ), whereas the plasma HDL-C levels was decreased in RA patients (WMD: $-5.21,95 \% \mathrm{Cl}:-7.69$ to -2.72 ). Furthermore, in the before-after studies, CEC of RA patients (SMD: $0.20,95 \%$ Cl: 0.03 to 0.38 ) was increased but the plasma HDL-C levels (WMD: $3.26,95 \% \mathrm{Cl}:-0.17$ to 6.69 ) was similar after anti-rheumatic treatment comparing to the baseline. In addition, stratified analysis suggested that DAS28 could be potential sources of heterogeneity for CEC. The funnel plot was relatively symmetric and was not suggestive of presence of publication bias.

Conclusion The current meta-analysis demonstrated a slight, nonsignificant, decrease in the CEC among RA patients as compared with healthy individuals, but it can be improved substantially following anti-rheumatic treatment. More large population studies evaluating the predictive role of CEC on cardiovascular risk in RA are needed.

\section{Introduction}

Rheumatoid arthritis (RA), a chronic polyarthritis autoimmune disease, causing arthrosis impairment and even leading to disability[1], affects approximately 0.3\%-1.0\% people around the world[2]. In addition to a heavy burden for both patient and society, those living with RA have a significantly reduced life expectancy. High risk of cardiovascular diseases (CVD) has been found in patients with RA compared with the general population[3]. RA inflammation increases arterial stiffness, changes lipid profile, and destabilizes plaque. Moreover, $60 \%$ excess mortality in RA is attributed to CVD[4], which is one of the most severe complications of RA and cannot be fully explained by traditional cardiovascular risk factors.

The high-density lipoprotein cholesterol (HDL-C) is known as the "good cholesterol" owing to its protective effects against CVD[5]. Numerous studies suggested that the plasma HDL-C levels were reduced in patients with RA compared to general individuals[6-8]. For example, a study noted that an average of 9\% drop in the plasma HDL-C level has been observed before the onset of symptoms among RA patients[9]. In addition, paradoxical associations among lowering lipid levels (i.e., total cholesterol (TC), low-density lipoprotein cholesterol (LDL-C) and HDL-C) and the ongoing risk of CVD were observed in patients with poorly controlled RA[10], while the initial reductions in these parameters have increased with anti-inflammatory treatment in patients with RA. So the European League Against Rheumatism (EULAR) indicated that the TC to HDL-C ratio would seem more appropriate to predict the CVD risk in RA[11]. However, recent clinical trials with HDL-C-raising therapies has shown rather than being protective, which still associated with high cardiovascular events risk[12-14]. Therefore, whether the concentrations of circulating HDL-C in patients with RA were altered even after anti-inflammatory treatment merits further investigation.

HDL exerts several key atheroprotective functions, including its capacity of reverse cholesterol transport, maintenance endothelial function, anti-inflammatory and inhibition of platelet aggregation[15]. Of these functions, reverse cholesterol transport (RCT) is a overriding process that promote excess plasma cholesterol from cell to the liver for catabolism ${ }^{6}$. Macrophages cholesterol efflux as the first critical step of RCT which is considered a key atheroprotective property[16]. The impaired HDL cholesterol efflux capacity (CEC) has been proven to increase the risk of CVD, which is independent of the plasma HDL-C levels[17,18]. However, the epidemiological data that have been performed to explore the association between CEC and RA was less convincing. Some of them have shown that the CEC was significantly reduced in RA patients compared to healthy controls, but others failed to reach the conclusions[19-28]. In 2012, Charles-schoeman et al. first reported that there was no significant difference in CEC among RA patients comparing to control groups, but which was inversely associated with high disease activity in RA patients[21]. Subsequently, another cross-sectional study published in 2014, which analyzed different CEC efflux pathways, demonstrated that ABCG1-mediated CEC was markedly impaired in RA group[19]. Furthermore, in 2015, another study also conducted by Ronda et al. found that CEC can be modified by methotrexate (MTX), but not MTX + adalimumab (ADA) in RA patients[25].

Therefore, to assess the conflicting results, we systematically reviewed and meta-analyzed the epidemiological evidence on the changes in CEC as well as HDL-C levels of RA patients.

\section{Methods}

\section{Literature Search and Selection Criteria}

We conduct this meta-analysis and written the manuscript according to the PRISMA guidelines. EMBASE, MEDLINE and Cochrane Library databases were searched for records reporting CEC in patients with RA. Searches were focused on human subjects with no restriction on language and published before 10 June 2020. To avoid missing any relevant studies, we also searched manually the bibliographies of identified studies and review papers. The following medical subject headings terms and keywords were used alone or in combination: "high density lipoprotein" or "HDL" or "HDL-C", "rheumatoid arthritis" or "RA", "high density lipoprotein function" or "cholesterol efflux capacity" or "CEC" or "HDL-mediated cholesterol efflux".

\section{Study selection}

Studies were initially assessed according to the following inclusion criteria: 1) we included all intervention trials and observational studies; 2) all subjects were older than 18 years of age; 3) RA patients who fulfilled 1987 or 2011 ACR criteria (fulfilling the American College of Rheumatology criteria) regardless of 
whether or not combination cardiovascular disease; 4) healthy control subjects without inflammatory conditions; 5) the outcomes of interest were CEC and HDL-C levels. Two researchers independently deleted duplicate records, screened the titles and abstracts to identify potentially relevant articles. We further performed a full-text screening to identify additional eligible studies. If data were duplicated in more than one study, we gave priority to include the articles with the largest datasets and populations.

\section{Data extraction and outcome measures}

Two reviewers independently extracted data on each eligible articles by using a standardized data collection form, including first author's name, publication year, study design, country, patient characteristics, sample size, gender proportion, CEC assay methods, plasma HDL-C levels, CEC, Disease Activity Score for 28 joints (DAS28), C-reactive protein (CRP), records of all medicines taken, length of follow-up and study outcome. For data reported as medians and ranges, which were converted to means and standard deviations using Hozo's approach[29]. Although some participants were followed-up for varying durations, data were extracted from the greatest time. If necessary, we contacted authors for additional data. Disagreements between reviewers were resolved by discussion among third author.

We firstly retrieved 2270 articles from the various databases. After deleting duplication and screening title and abstract, 2235 articles were further excluded. The remaining 35 articles were reviewed with full text according to inclusion criterion. Eventually, only 11 articles were included. 26 papers were excluded due to the following reasons: 6 studies did not assess the outcome of CEC; 3 were reviews; 10 were abstracts without published full-text; 6 were animal experimentation. For the rest listed of studies, there were totally 6 observational studies and 5 intervention studies. The whole screening flow chart was posted in Fig 1.

\section{Quality and risk Assessment}

Two quality ratings scales assessed the methodological quality of studies. First, the quality of observational study was evaluated by the Newcastle-Ottawa Scale (NOS). According to the guidelines, three aspects were assessed: selection, comparability and exposure. The score of 1-4 were defined as low quality, and 5-9 as high quality respectively. Second, the Downs and Black scale (D\&B) was adopted to analyze the bias risk of non-randomized and randomized studies from a list of 27 criteria. The last question about power was replaced by modification version, that was published in previous systematic reviews[30]. The score of 1-14 were defined as low quality, and 15-32 as high quality respectively.

\section{Statistical Analysis}

Data from observational studies and intervention studies were analyzed respectively. Mean and SD were commonly used to evaluate the changes of CEC and plasma HDL-C levels in RA patients. For continuous variables, the standardized and weighted mean differences (SMD \& WMD) as well as corresponding 95\% confidence intervals $\left(\mathrm{Cl}_{\mathrm{s}}\right)$ were calculated. For the intervention studies, baseline data was collected as the control group and data from the end of the treatment phase as the experimental group. Heterogeneity across eligible studies were obtained by using the Cochran's $Q$ test. We further performed subgroup analysis to explore the sources of heterogeneity. By comparing the random and fixed effects models, we subsequently conducted a sensitivity analysis, and the influence of each individual study on the overall outcome was accessed by excluding one study at a time. The risk of publication bias was observed by visual graph of funnel plots and further quantified using Egger's test, where $P<0.1$ indicates potential publication bias[31,32]. All analyses were performed using STATA version 14.0 (StataCorp. 2015. Stata Statistical Software: Release 14. College Station, TX: StataCorp LP.) and Review Manager Version 5.3 (Copenhagen: The Nordic Cochrane Centre, The Cochrane Collaboration, 2014).

\section{Results}

\section{Literature search results and study characteristics}

Table 1 and Table 2 showed the main characteristic of the included studies. These 11 articles were published during 2012 to 2019 . Of which 7 studies were conducted in the USA, 3 in UK, and 1 in Spain. The sample size of those studies ranged from 36 to 401 , several of them with sample sizes range 50 to 100 were considered modest, and 3 other studies with a large sample size ( $>100)$. The average age of participants ranged from 42 to 65 years. Cases and controls were matched by gender, age and body mass index (BMI) in case-control study. According to the NOS and D\&B standard criteria, the quality scores of the 6 observational studies were 5 to 8 , which were all considered as moderate and high-quality. Moreover, the quality scores of the intervention studies ranged from 11 to 17 , and 2 studies were considered as high-quality and 3 as low-quality studies. Most observational studies did not report the non-response rate, and the D\&B scores showed weak external validity of intervention studies.

\section{The main outcome: changes in CEC and HDL-C levels among RA patients}

Observational studies: 5 observational studies reported the CEC in both case and control groups with a total of 809 participants. Pooled results showed that the CEC of RA patients didn't decrease significantly comparing with the healthy controls (SMD: $-0.22,95 \% \mathrm{Cl}:-0.65$ to $0.20 ; P=86 \%, P$ for heterogeneity $<$ 0.001). In addition, 6 studies with 845 subjects measuring the plasma HDL-C levels showed the patients with RA had a lower HDL-C levels (WMD: -5.21 , 95\% Cl: -7.69 to -2.72$)$, with a nonsignificant heterogeneity $(R=54 \%, P$ for heterogeneity $=0.050)($ Fig 2$)$. Besides, the plasma levels of TC (total cholesterol) and LDL were both significantly altered in two groups, except for TG (triglycerides) (see Additional file 1).

Before-after studies: 5 studies that include 8 trials revealed a statistically significant elevation of CEC in RA patients who had taken anti-rheumatic medications comparing to the baseline (SMD: $0.20,95 \% \mathrm{Cl}$ : 0.03 to $0.38 ; P=0 \%, P$ for heterogeneity $=0.650$ ). In addition, only 4 studies were suitable to analyze the change of HDL-C levels in the intervention studies. In contrast, the plasma HDL-C levels were slightly higher in RA patients after anti-rheumatic drug treatment comparing to the baseline, but the difference did not reach the level of significance. (WMD: $3.26,95 \% \mathrm{Cl}$ : -0.17 to 6.69 ). The heterogeneity was 
quite high $(R=0 \%, P$ for heterogeneity $=0.640)(F i g)$. Other lipid parameters such as LDL, TC and TG were not significantly different between baseline and follow-up (see Additional file 2). 5 studies reported CRP levels as a continuous variable. Accordingly, in observational studies, the pooled analysis data showed that the CRP levels increased in RA patients compared to the healthy controls (SMD: $1.25,95 \% \mathrm{Cl}: 0.33$ to $2.16 ; P=96 \%, P$ for heterogeneity $<0.001$ ). However, the CRP levels were significantly reduced after anti-rheumatic drug therapy among the intervention studies (SMD: $-0.46,95 \%$ Cl: -0.71 to $-0.21 ; P=0 \%, P$ for heterogeneity $=0.830$ ).

\section{Subgroups and sensitivity analysis}

Our results found that DAS28 had a significant impact on the heterogeneity of CEC $(R=22.6 \%)$. In addition, subgroup analysis stratified by BMI and age showed that the heterogeneity of HDL-C levels was significantly decreased $\left(R=36.8 \% ; R^{2}=19.6 \%\right.$, respectively) (see Additional file 3 ). The sensitivity analysis showed that the overall effect size of CEC and CRP obtained using fixed-effect and random-effects models were identical and no individual study significantly affected the pooled results. The results in the random effects and fixed effects models as well as the subgroup analysis were similar. After further excluding a signal article[18], low heterogeneity was found after remerging $(P=36.8 \%)$, and the results of random effects models were consistent with the results of fixed effects model (fixed effects model: WMD: $-6.07,95 \% \mathrm{Cl}:-8.67$ to -3.64 ; random effects model: WMD: $-5.45,95 \%$ Cl: -9.40 to -1.51 ) (see Additional file 4).

\section{Publication bias}

We performed funnel plots for CEC, HDL and CRP to assess publication bias (Fig 4). The presence of publication bias was also evaluated by using the Begg and Egger tests. Currently, the publication bias of CEC, HDL-C and CRP were calculated $\left(P_{\mathrm{CEC}}=0.106 ; P_{\mathrm{HDL}-\mathrm{C}}=0.342 ; P_{\mathrm{CRP}}=0.721\right)$ according to funnel plot and Egger's test. However, statistical power of publication bias was low due to a small number of studies.

\section{Discussion}

To the best of our knowledge, this is the first systematic review and meta-analysis to simultaneously evaluate whether the CEC and plasma HDL-C levels were decreased in RA or altered by drug therapy. The results did not show any significant change in the HDL-meditated CEC, while the plasma HDL-C levels decreased significantly in patients with RA compared to the healthy subjects. In addition, stratified analysis did not influence the tendency of CEC and HDL-C levels in RA. However, it is interesting that in the intervention study, the elevated CEC was seen in RA patients taking their medications, followed by reduction of the CRP levels compared to the baselines. These findings suggested that inhibition of inflammation might improve HDL-mediated CEC. Although the plasma HDL-C levels were slightly increased after RA treatment, the difference did not reach the level of significance, which indirectly indicated that CEC is more sensitive than HDL-C. Several potential mechanisms can thus be proposed to explain the changes of CEC, plasma HDL-C and CRP levels in patients with RA.

RA is an autoimmunity disease associated with chronic inflammation, which might lead to multiple changes in HDL structure and alterations in HDL function[33]. First, most patients with RA have elevated levels of certain proinflammatory cytokines, including CRP, interleukin-6 in their blood. Many studies have indicated that HDL-C levels were dramatically reduced during inflammation, and one possible mechanism underlying these changes associated with the live phospholipid transfer protein (PLTP) expression. For example, Audo et al. showed that PLTP is highly expressed in the joints of RA patients and may directly trigger inflammation[34]. Furthermore, Jiang et al. demonstrated that the reduced plasma PLTP activity results in markedly decreased plasma HDL lipid and apolipoproteins, indicating the importance of the transfer of triglyceride-rich lipoproteins surface components in maintaining HDL levels[35]. In our meta-analysis, we found that the plasma HDL-C levels were significantly decreased in the RA patients compared to the healthy groups, which suggested that the inflammatory status and severity of patients with RA was inversely related to the plasma HDL-C levels. However, the plasma HDL-C levels did not change significantly after RA treatment.

Second, substantial evidence has now accumulated, suggesting that a high inflammation status would limit capacity for HDL to promote cholesterol efflux from of macrophages[36]. McGillicuddy et al. conducted a study in mice found that acute inflammation has a broad and integrated impact on RCT in vivo, attenuating several steps including macrophage cholesterol efflux, HDL acceptor function, and hepatic to bile/fecal cholesterol elimination[37]. Thus, which suggested that the inflammatory status in patients with RA not only affect the rate of cholesterol efflux but also other steps in RCT. Surprisingly, in our metaanalysis we found that the CEC was not significantly decreased different between the RA patients and control groups. Possibly, routinely measured baseline CEC in RA patients may not accurate due to the fluctuations of inflammatory status. Meanwhile, despite several studies used a few different approaches to measure CEC, and even some of them might under estimate the actual cholesterol efflux. However, large quantity of population-based evidences demonstrated that CEC was a sensitive predictor of the risk of CVD, independent of circulating HDL-C concentrations[38,39]. In addition, we found that the CEC was significantly increased in patients with RA after medicine care, followed by reduction of the CRP levels compared to the baselines, while the HDL-C levels did not significantly alter after anti-rheumatoid treatment. These findings indirectly indicated that CEC might serve as more sensitive indicator than HDL-C levels in prevention of CVD in RA. Generally, effectively control inflammation in RA might help to improve the function of HDL. Some possible mechanisms have been proposed to determine the specific role of increasing CEC for prevention of CVD in patients with RA in present evidences. Further larger-scale population-based studies and experimental researches are needed to confirm the fundamental roles and mechanisms.

Heterogeneity poses an important challenge in conducting and interpreting the results of meta-analyses[40]. We conducted subgroup analysis to evaluate the heterogeneity of CEC and HDL-C. The analysis results have found that DAS 28 is the source of statistic heterogeneity. For the HDL levels, BMI and age, not DAS28, were the sources of heterogeneity. Sensitivity analysis were performed to enhance the robustness and reliability of results. The effect size of HDL-C levels was not significantly different in random model, when we included a study conducted by Ronda et al[25], which may due to the younger age and prohibition of taking statins in RA patients, both could leading to markedly raise of the plasma HDL-C levels. Furthermore, nearly half of the included studies were case-control studies, which have comparatively low quality. Given that the possible recall bias and selection bias in case-control studies, more large-scale 
prospective cohort studies with full adjustment for potential confounding factors are urgently needed to confirm the inverse association between CEC and CRP levels.

Several potential limitations should be taken into consideration. First, the quality of meta and pooled analysis largely depended on the quality of the original studies. Among the studies included, there were six observational studies, which were susceptible to selection and recall bias. Second, a substantial heterogeneity have reported in this meta analysis. Therefore, sensitivity analyses were conducted to confirm the stability of the results. Third, currently there was no established gold-standard for ex-vivo CEC assays. Besides, the rate of cholesterol efflux was expressed in various form, leading to slight difference between CEC values. Thus, to minimize the variation, we calculated standardized effect sizes. Fourth, the small number of included studies could limit the ability to interpret the funnel plot.

\section{Conclusion}

In summary, our meta-analysis observed a slight, nonsignificant, decrease in the CEC among RA patients as compared with healthy individuals but it can be improved substantially following anti-rheumatic treatment. In contrast, the plasma HDL-C levels were not associated with risk of RA. Overall, the CEC of RA patients could be improved through earlier control of inflammation and anti-rheumatic treatment. Future large population-based and long-term studies should further elucidate the changes in CEC of RA patients and assess the function in preventing CVD.

\section{Abbreviations}

RA Rheumatoid arthritis

CVD Cardiovascular diseases

HDL-C High-density lipoprotein cholesterol

TC Total cholesterol

LDL-C Low-density lipoprotein cholesterol

TG Triglycerides

EULAR The European League Against Rheumatism

RCT Reverse cholesterol transport

MTX Methotrexate

ADA Adalimumab

PLTP Phospholipid transfer protein

DAS28 Disease Activity Score for 28 joints

CRP C-reactive protein

D\&B Downs and Black scale

NOS Newcastle-Ottawa Scale

SMD Standardized mean differences

WMD Weighted mean differences

Cls 95\% 95\% Confidence intervals

BMI Body mass index

\section{Declarations}

\section{Availability of data and materials}

Not applicable.

\section{Authors' contribution}

Study concept and design: CQ-L and JH. Data extraction and analysis: B-BX, YL and TL. Manuscript drafting: B-BX and CQ-L. All authors were involved in data analysis, drafting the article or revising it critically for important intellectual content, and all authors approved the final version to be published.

\section{Ethics approval and consent to participate}


Not applicable.

\section{Consent for publication}

Not applicable.

\section{Conflict of interest}

The authors declare that they have no competing interest.

\section{Publisher's Note}

Springer Nature remains neutral with regard to jurisdictional claims in published maps and institutional affiliations.

\section{Funding}

This work was supported by the National Natural Science Foundation of China (No.81903294), Guangdong Medical Science and Technology Research Fund Project (No. A2018342), Fundamental Research Funds for the Central Universities, Natural Science Foundation of Guangdong Province (NO. 2018030310412) , and Project from Jinan University (No. 21617352).

\section{References}

1. Sangha O. Epidemiology of rheumatic diseases. Rheumatology (Oxford) 2000;39:3-12.

2. Woolf AD, Pfleger B. Burden of major musculoskeletal conditions. Bull World Health Organ 2003;81:646-56.

3. Symmons DP, Gabriel SE. Epidemiology of CVD in rheumatic disease, with a focus on RA and SLE. Nat Rev Rheumatol 2011;7:399-408.

4. Meune C, Touze E, Trinquart L, Allanore Y. Trends in cardiovascular mortality in patients with rheumatoid arthritis over 50 years: a systematic review and meta-analysis of cohort studies. Rheumatology (Oxford) 2009;48:1309-1313.

5. Rader DJ, Hovingh GK. HDL and cardiovascular disease. Lancet 2014;384:618-25.

6. Alsalawy AM, Fathi Al, Kamel RA, Ewis I. Correlation between serum osteoprotegerin and atherosclerotic vascular disorders in rheumatoid arthritis patients. The Egyptian Rheumatologist 2012;34:35-42.

7. Vergeer M, Holleboom AG, Kastelein JJ, Kuivenhoven JA. The HDL hypothesis: does high-density lipoprotein protect from atherosclerosis? J Lipid Res 2010;51:2058-73.

8. Kim J-Y, Lee E-Y, Park JK, Song YW, Kim J-R, Cho KH. Patients with Rheumatoid Arthritis Show Altered Lipoprotein Profiles with Dysfunctional HighDensity Lipoproteins that Can Exacerbate Inflammatory and Atherogenic Process. PLoS One 2016;11:e0164564.

9. Nurmohamed MT. Atherogenic lipid profiles and its management in patients with rheumatoid arthritis. Vasc Health Risk Manag 2007;3:845-52.

10. Myasoedova E, Crowson CS, Kremers HM, Roger VL, Fitz-Gibbon PD, Therneau TM, et al. Lipid paradox in rheumatoid arthritis: The impact of serum lipid measures and systemic inflammation on the risk of cardiovascular disease. Ann Rheum Dis 2011;70:482-7.

11. Chodara AM, Wattiaux A, Bartels CM. Managing Cardiovascular Disease Risk in Rheumatoid Arthritis: Clinical Updates and Three Strategic Approaches. Curr Rheumatol Rep 2017;19:16.

12. Boden WE, Probstfield JL, Anderson T, Chaitman BR, Desvignes-Nickens P, Desvignes-Nickens P, et al. Niacin in patients with low HDL cholesterol levels receiving intensive statin therapy N Engl J Med 2011;365:2255-2267.

13. Taylor AJ. Given the ENHANCE trial results, ezetimibe is still unproven. Cleve Clin J Med 2008;75:497-506.

14. Brown BG, Taylor AJ. Does ENHANCE diminish confidence in lowering LDL or in ezetimibe?. N Engl J Med 2008;358:1504-1507.

15. Rosenson RS, Brewer HB Jr, Davidson WS, Fayad ZA, Fuster V, Goldstein J, et al. Cholesterol efflux and atheroprotection: advancing the concept of reverse cholesterol transport. Circulation 2012;125:1905-1919.

16. Jeong SJ, Lee MN, Oh GT. The Role of Macrophage Lipophagy in Reverse Cholesterol Transport. Endocrinol Metab (Seoul) 2017;32:41-46.

17. Kosmas CE, Martinez I, Sourlas A, Bouza KV, Campos FN, Torres V, et al. High-density lipoprotein (HDL) functionality and its relevance to atherosclerotic cardiovascular disease. Drugs Context 2018;7:212525.

18. Rohatgi A, Khera A, Berry JD, Givens EG, Ayers CR, Wedin KE, et al. HDL cholesterol efflux capacity and incident cardiovascular events. N Engl J Med 2014;371:2383-2393.

19. Ronda N, Favari E, Borghi MO, Ingegnoli F, Gerosa M, Chighizola C, et al. Impaired serum cholesterol efflux capacity in rheumatoid arthritis and systemic lupus erythematosus. Ann Rheum Dis 2014;73:609-15.

20. Vivekanandan-Giri A, Slocum JL, Byun J, Tang C, Sands RL, Gillespie BW, et al. High density lipoprotein is targeted for oxidation by myeloperoxidase in rheumatoid arthritis. Ann Rheum Dis 2013;72:1725-31.

21. Charles-Schoeman C, Lee YY, Grijalva V, Amjadi S, FitzGerald J, Olmos JM, et al. Cholesterol efflux by high density lipoproteins is impaired in patients with active rheumatoid arthritis. Ann Rheum Dis 2012;71:1157-62.

22. O'Neill F, Charakida M, Topham E, McLoughlin E, Patel N, Sutill E, et al. Anti-inflammatory treatment improves high-density lipoprotein function in rheumatoid arthritis. Heart 2017;103:766-73. 
23. Ormseth MJ, Yancey PG, Yamamoto S, Oeser AM, Gebretsadik T, Shintani A, et al. Net cholesterol efflux capacity of HDL enriched serum and coronary atherosclerosis in rheumatoid arthritis. IJC Metab Endocr 2016;13:6-11.

24. Tejera-Segura B, Macia-Diaz M, Machado JD, de Vera-Gonzalez A, Garcia-Dopico JA, OlmosNJM, et al. HDL cholesterol efflux capacity in rheumatoid arthritis patients: contributing factors and relationship with subclinical atherosclerosis. Arthritis Res Ther 2017;19:113.

25. Ronda N, Greco D, Adorni MP, Zimetti F, Favari E, Hjeltnes G, et al. Newly identified antiatherosclerotic activity of methotrexate and adalimumab: complementary effects on lipoprotein function and macrophage cholesterol metabolism. Arthritis Rheumatol 2015;67:1155-1164.

26. Liao KP, Playford MP, Frits M, Coblyn JS, lannaccone C, Weinblatt ME, et al. The association between reduction in inflammation and changes in lipoprotein levels and HDL cholesterol efflux capacity in rheumatoid arthritis. J Am Heart Assoc 2015;4:e001588.

27. Ormseth MJ, Yancey PG, Solus JF, Bridges SL Jr, Curtis JR, Machado JD, et al. Effect of drug therapy on net cholesterol efflux capacity of high-density lipoprotein-enriched serum in rheumatoid arthritis. Arthritis Rheumatol 2016;68:2099-105.

28. Ferraz-Amaro I, Hernández-Hernández MV, Tejera-Segura B, Delgado-Frías E, Macía-Díaz M, Linton MF, et al. Effect of IL-6 Receptor Blockade on Proprotein Convertase Subtilisin/Kexin Type-9 and Cholesterol Efflux Capacity in Rheumatoid Arthritis Patients. Horm Metab Res 2019;51:200-209.

29. Hozo SP, Djulbegovic B, Hozo I. Estimating the mean and variance from the median, range, and the size of a sample. BMC Med Res Methodol $2005 ; 5: 13$.

30. Sohanpal R, Hooper R, Hames R, Priebe S, Taylor S. Reporting participation rates in studies of non-pharmacological interventions for patients with chronic obstructive pulmonary disease: a systematic review. Syst Rev 2012;1:66.

31. Begg CB, Mazumdar M. Operating characteristics of a rank correlation test for publication bias. Biometrics 1994;50:1088-101.

32. Egger M, Davey Smith G, Schneider M, Minder C. Bias in meta-analysis detected by a simple, graphical test. Bmj 1997;315:629-34.

33. Feingold KR, Grunfeld C. Effect of inflammation on HDL structure and function. Curr Opin Lipidol 2016;27:521-530.

34. Audo R, Deckert V, Daien Cl, Che H, Elhmioui J, Lemaire S, et al. PhosphoLipid transfer protein (PLTP) exerts a direct pro-inflammatory effect on rheumatoid arthritis (RA) fibroblasts-like-synoviocytes (FLS) independently of its lipid transfer activity. PLoS One 2018;13:e0193815.

35. Jiang XC, Bruce C, Mar J, Lin M, Ji Y, Francone OL, et al. Targeted mutation of plasma phospholipid transfer protein gene markedly reduces high-density lipoprotein levels. J Clin Invest 1999;103:907-14.

36. Malik P, Berisha SZ, Santore J, Agatisa-Boyle C, Brubaker G, Smith JD. Zymosan-mediated inflammation impairs in vivo reverse cholesterol transport. J Lipid Res 2011;52:951-

37. McGillicuddy FC, de la Llera Moya M, Hinkle CC, Joshi MR, Chiquoine EH, Billheimer JT, et al. Inflammation impairs reverse cholesterol transport in vivo. Circulation 2009;119:1135-45.

38. Anastasius M, Kockx M, Jessup W, Sullivan D, Rye KA, Kritharides L. Cholesterol efflux capacity: An introduction for clinicians. Am Heart J 2016;180:54-63.

39. Mortensen MB, Afzal S, Nordestgaard BG, Falk E. The high-density lipoprotein-adjusted SCORE model worsens SCORE-based risk classification in a contemporary population of 30,824 Europeans: the Copenhagen General Population Study. Eur Heart J 2015;36:2446-2453.

40. Threapleton DE, Greenwood DC, Evans CE, Cleghorn CL, Nykjaer C, Woodhead C, et al. Dietary fibre intake and risk of cardiovascular disease: systematic review and meta-analysis. BMJ 2013;347:f6879.

\section{Tables}

Table 1. Characteristics of observational studies in this systematic review and meta-analysis

\begin{tabular}{|c|c|c|c|c|c|c|c|c|}
\hline Authors & Country & $\begin{array}{l}\text { Study } \\
\text { type }\end{array}$ & $\begin{array}{l}\text { Subjects } \\
\text { (female, } \\
\% \text { ) }\end{array}$ & $\begin{array}{l}\text { Duration }^{a} \\
\text { (month) }\end{array}$ & $\begin{array}{l}\text { Assay method } \\
\text { of CEC }\end{array}$ & $\begin{array}{l}\text { Labeled- } \\
\text { cholesterol }\end{array}$ & $\begin{array}{l}\text { Quality } \\
\text { score }\end{array}$ & Outcome summary \\
\hline Ronda et al., 2013 ${ }^{[18]}$ & USA & $\begin{array}{l}\text { Case- } \\
\text { control }\end{array}$ & $\begin{array}{l}52 \\
(86.7)\end{array}$ & $N / A^{c}$ & $\begin{array}{l}\text { J774, Fu5AH, } \\
\text { CHO-k1 to ApoB- } \\
\text { depleted serum }\end{array}$ & $\begin{array}{l}{ }^{3} \mathrm{H}- \\
\text { cholesterol }\end{array}$ & 5 & $\mathrm{CEC}^{\mathrm{b}}$ is impaired in RA \\
\hline $\begin{array}{l}\text { Vivekanandan-Giri et } \\
\text { al., } 2013^{[19]}\end{array}$ & USA & $\begin{array}{l}\text { Case- } \\
\text { control }\end{array}$ & $\begin{array}{l}34 \\
(58.6)\end{array}$ & 4.0 & $\begin{array}{l}\text { J774 to ApoB- } \\
\text { depleted serum }\end{array}$ & $\begin{array}{l}{ }^{3} \mathrm{H}- \\
\text { cholesterol }\end{array}$ & 5 & $\begin{array}{l}\text { CEC was diminished in RA } \\
\text { patients }\end{array}$ \\
\hline $\begin{array}{l}\text { Charles-Schoeman et } \\
\text { al., } 2015^{[20]}\end{array}$ & USA & $\begin{array}{l}\text { Case- } \\
\text { control }\end{array}$ & $\begin{array}{l}66 \\
(82.0)\end{array}$ & 12.8 & $\begin{array}{l}\text { RAW264.7 to } \\
\text { isolated } \mathrm{HDL}\end{array}$ & $\begin{array}{l}{ }^{3} \mathrm{H}- \\
\text { cholesterol }\end{array}$ & 9 & $\begin{array}{l}\text { no difference of CEC between } \\
\text { RA patients and controls }\end{array}$ \\
\hline O'Neill et al., 2016 ${ }^{[21]}$ & UK & $\begin{array}{l}\text { Case- } \\
\text { control }\end{array}$ & $\begin{array}{l}22 \\
(73.0)\end{array}$ & 5.0 & $\begin{array}{l}\text { J774 to ApoB- } \\
\text { depleted serum }\end{array}$ & $\begin{array}{l}{ }^{3} \mathrm{H}- \\
\text { cholesterol }\end{array}$ & 7 & - \\
\hline Ormseth et al.,2016 ${ }^{[22]}$ & USA & $\begin{array}{l}\text { Case- } \\
\text { control }\end{array}$ & $\begin{array}{l}144 \\
(68.6)\end{array}$ & 3.9 & $\begin{array}{l}\text { THP-1 to ApoB- } \\
\text { depleted serum }\end{array}$ & $\begin{array}{l}{ }^{3} \mathrm{H}- \\
\text { cholesterol }\end{array}$ & 6 & $\begin{array}{l}\text { CEC is not significantly altered } \\
\text { in RA patients }\end{array}$ \\
\hline $\begin{array}{l}\text { Tejera-Segura } \\
\text { et al., 2017 }\end{array}$ & Spain & $\begin{array}{l}\text { Cross- } \\
\text { sectional }\end{array}$ & $\begin{array}{l}295 \\
(73.6)\end{array}$ & 7.0 & $\begin{array}{l}\text { J774 to } \\
\text { ApoB-depleted } \\
\text { serum }\end{array}$ & $\begin{array}{l}\text { BODIPY- } \\
\text { cholesterol }\end{array}$ & 7 & $\begin{array}{l}\text { CEC is not significantly altered } \\
\text { in RA patients }\end{array}$ \\
\hline
\end{tabular}

Data are presented as median (interquartile range) or mean \pm SD unless otherwise indicated; ${ }^{a}$ duration of RA; ${ }^{b}$ cholesterol efflux capacity; ${ }^{c}$ not available. 
Table 2. Characteristics of interventional studies in this systematic review and meta-analysis

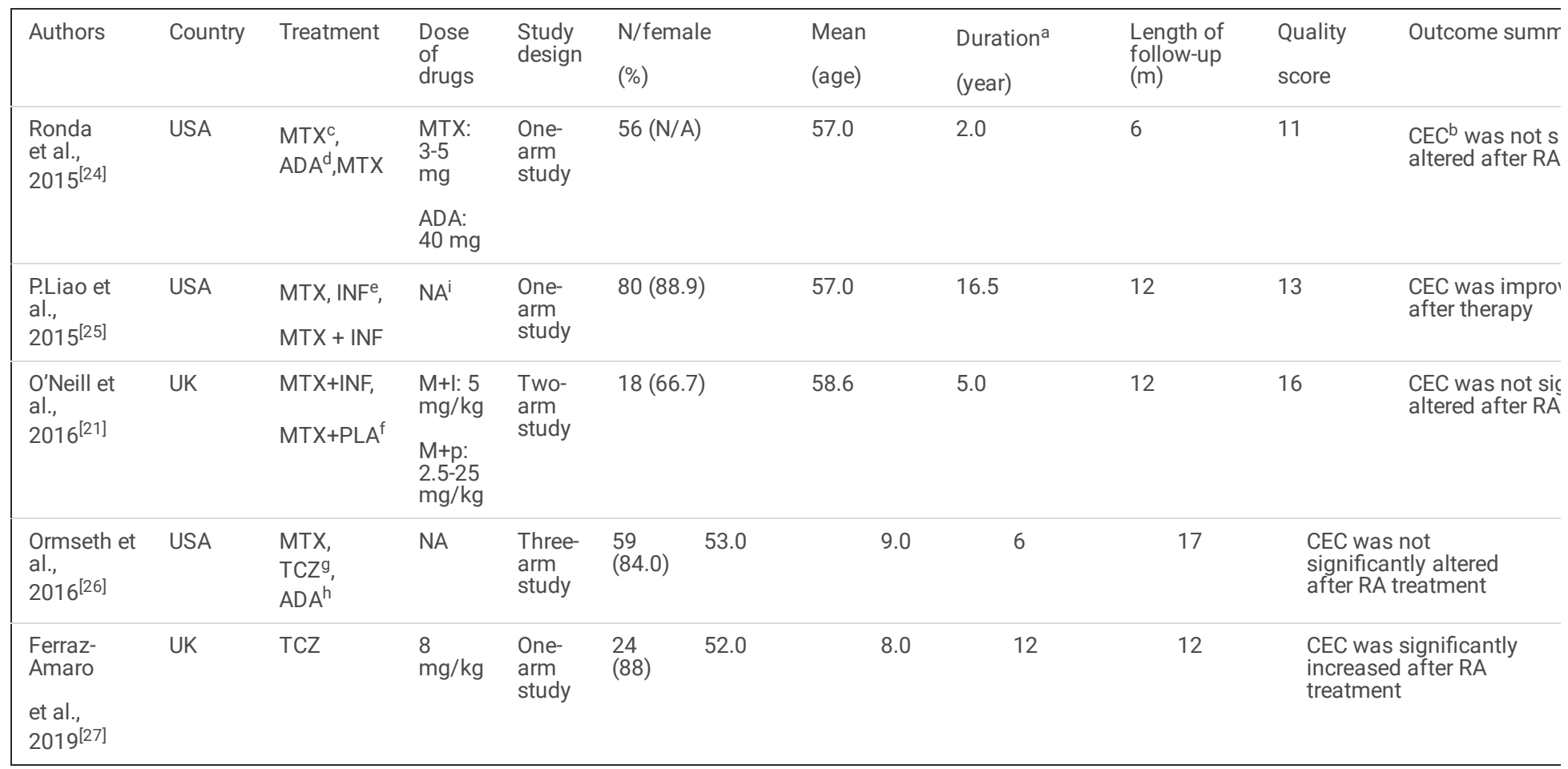

Data are presented as median (interquartile range) or mean \pm SD unless otherwise indicated; ${ }^{a}$ duration of RA; ${ }^{b}$ cholesterol efflux capacity; ${ }^{c}$ methotrexate; ${ }^{d}$ adalimumab; ${ }^{\mathrm{e}}$ inflixximab; ${ }^{\mathrm{f}}$ placebo; ${ }^{\mathrm{g}}$ tocilizumab; ${ }^{\mathrm{h}}$ adalimumab; ${ }^{\mathrm{i}}$ not available.

\section{Figures}




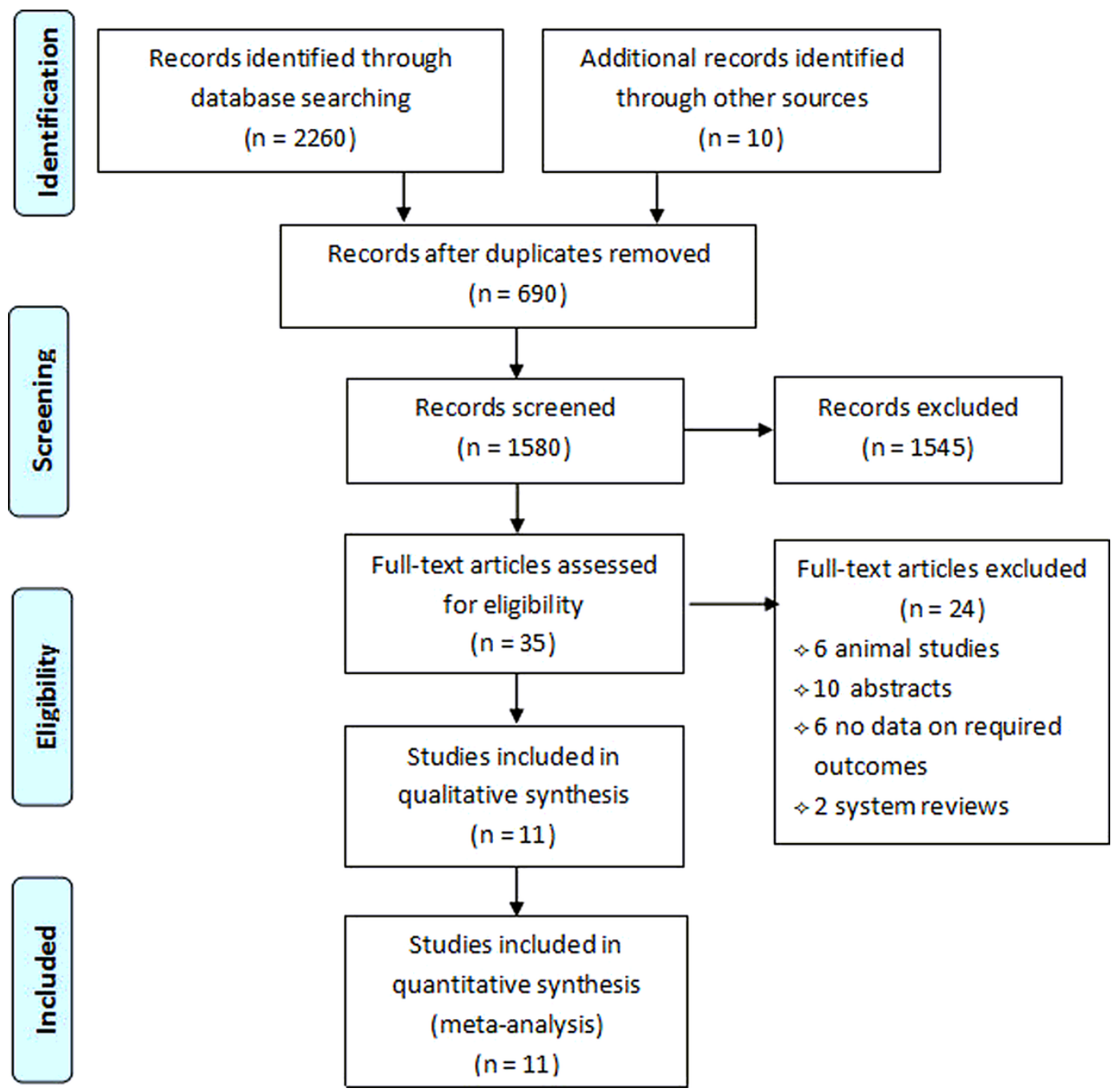

Figure 1

Flow diagram of selection process in the meta-analysis. 


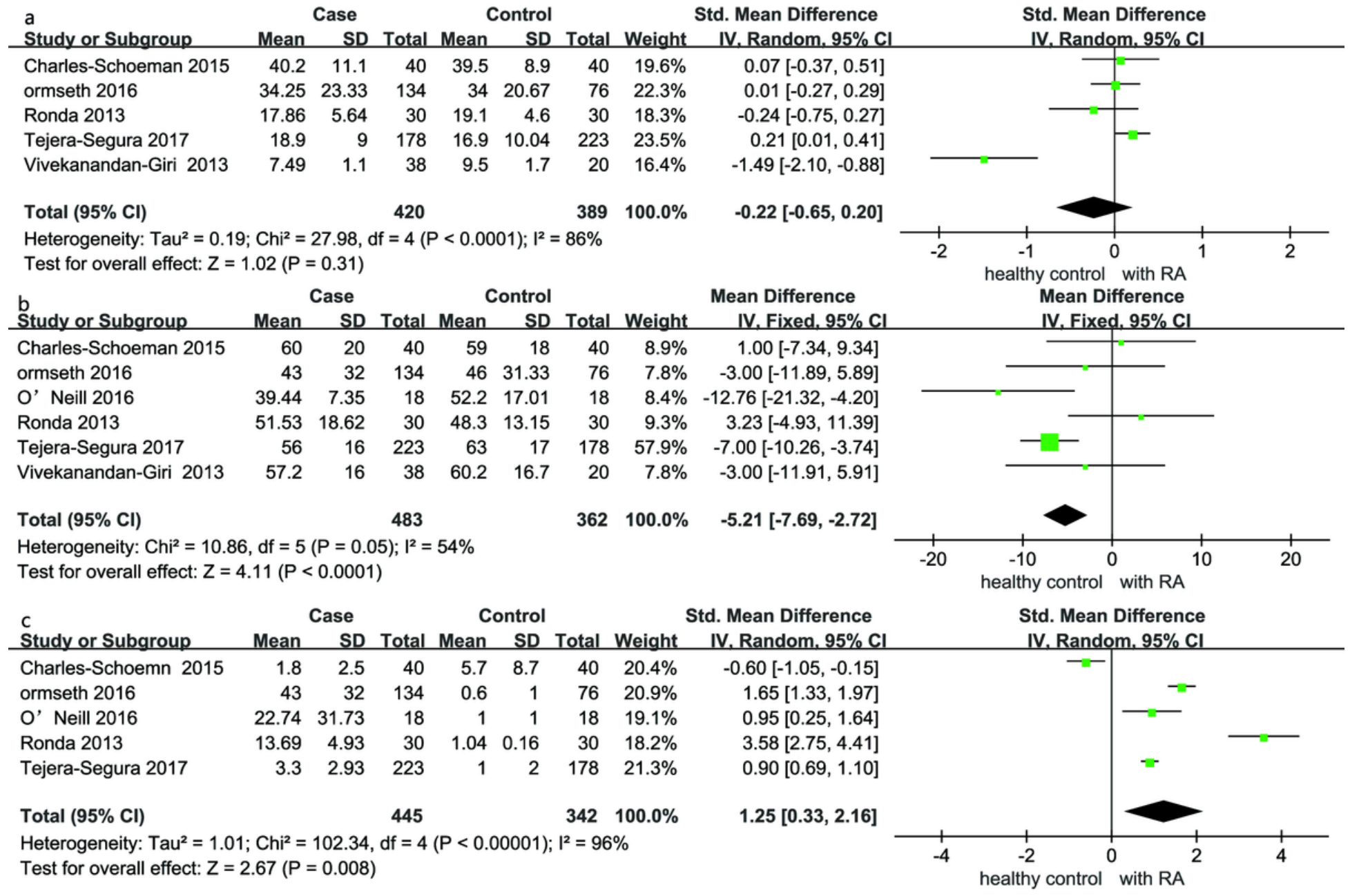

Figure 2

Forest plot of the plasma levels of CEC (a), high-density lipoprotein (b) and C-reaction protein (c) for patients with RA versus control group in observational study. 


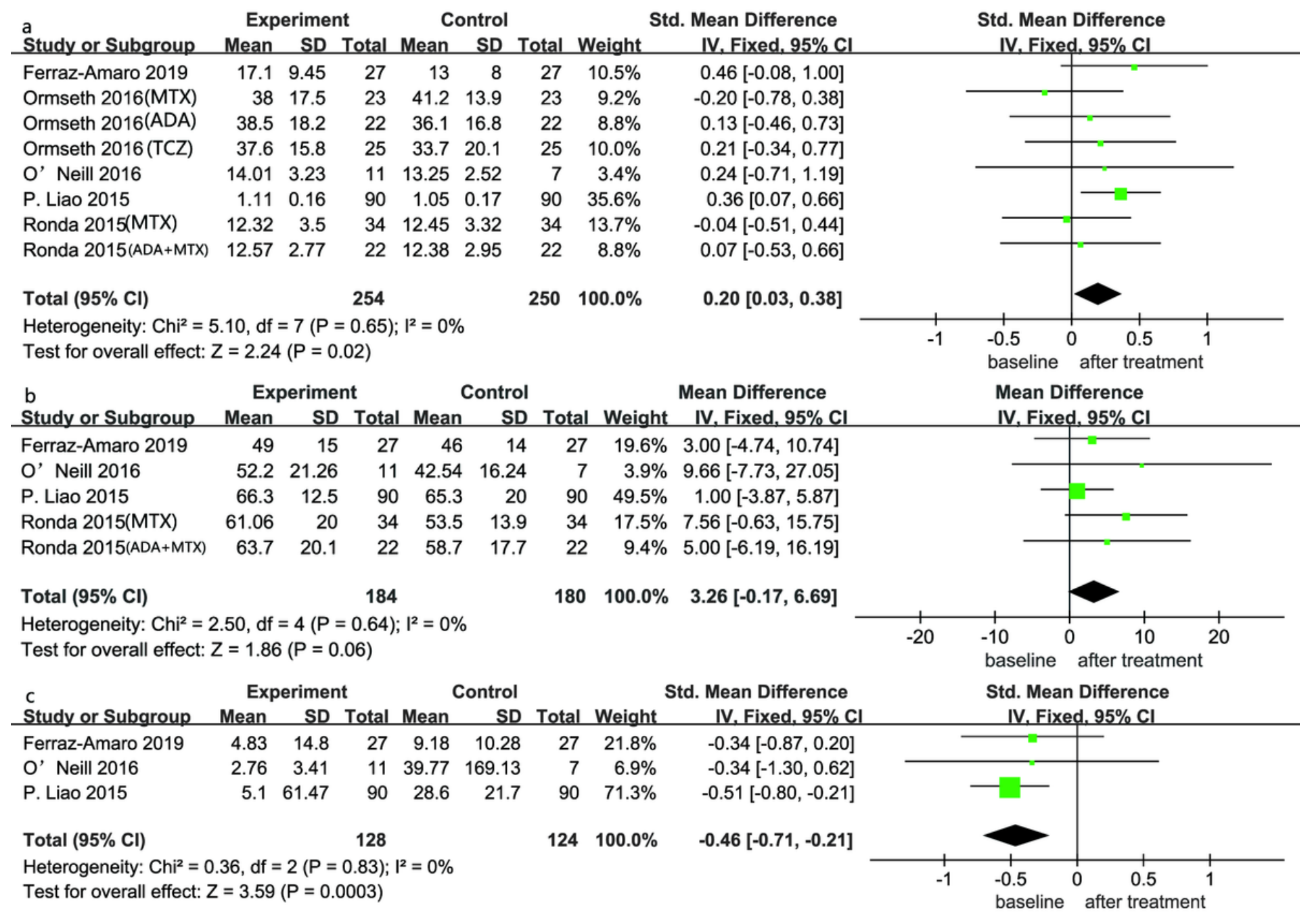

Figure 3

Forest plot of the plasma levels of CEC (a), high-density lipoprotein (b) and C-reaction protein (c) for patients with RA and control group in before-after studies
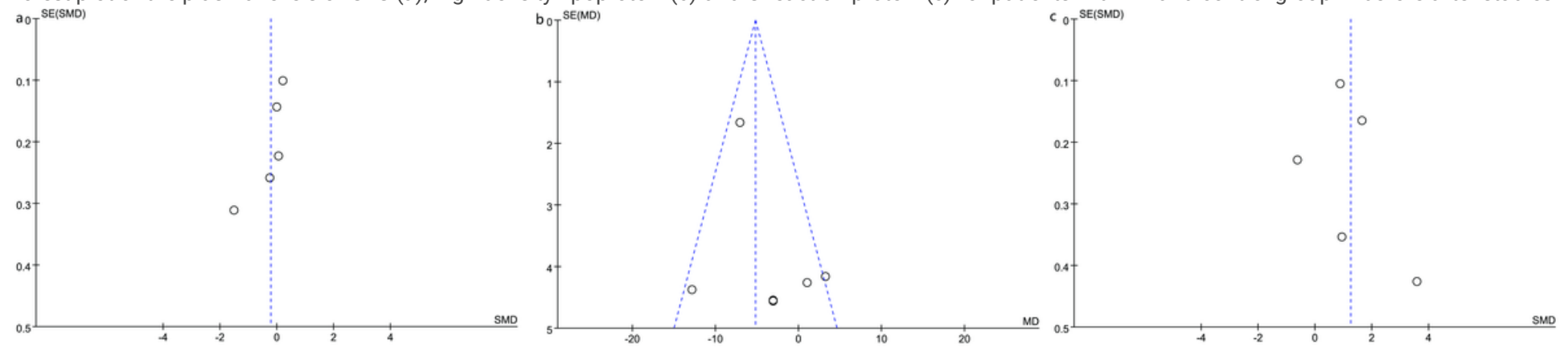

Figure 4

Funnel plots evaluating the pooled estimates for the plasma levels of CEC (a), high-density lipoprotein (b) and C-reaction protein (c) among patients with RA.

\section{Supplementary Files}

This is a list of supplementary files associated with this preprint. Click to download.

- Additionalfile4.docx

- Additionalfile3.docx

- Additionalfile2.tif

- Additionalfile1.tif 